\title{
Determination of the evaporation coefficient of $D_{2} O$
}

\author{
W. S. Drisdell ${ }^{1,2}$, C. D. Cappa ${ }^{3}$, J. D. Smith ${ }^{2}$, R. J. Saykally ${ }^{1,2}$, and R. C. Cohen ${ }^{1,4}$ \\ ${ }^{1}$ Department of Chemistry, UC Berkeley, Berkeley, CA, USA \\ ${ }^{2}$ Chemical Sciences Division, Lawrence Berkeley National Laboratory, Berkeley, CA, 94720, USA \\ ${ }^{3}$ Department of Civil and Environmental Engineering, UC Davis, Davis, CA, USA \\ ${ }^{4}$ Department of Earth and Planetary Science, UC Berkeley, Berkeley, CA, USA
}

Received: 26 March 2008 - Published in Atmos. Chem. Phys. Discuss.: 9 May 2008

Revised: 7 October 2008 - Accepted: 7 October 2008 - Published: 20 November 2008

\begin{abstract}
The evaporation rate of $\mathrm{D}_{2} \mathrm{O}$ has been determined by Raman thermometry of a droplet train $(12-15 \mu \mathrm{m}$ diameter) injected into vacuum $\left(\sim 10^{-5}\right.$ torr $)$. The cooling rate measured as a function of time in vacuum was fit to a model that accounts for temperature gradients between the surface and the core of the droplets, yielding an evaporation coefficient $\left(\gamma_{e}\right)$ of $0.57 \pm 0.06$. This is nearly identical to that found for $\mathrm{H}_{2} \mathrm{O}(0.62 \pm 0.09)$ using the same experimental method and model, and indicates the existence of a kinetic barrier to evaporation. The application of a recently developed transition-state theory (TST) model suggests that the kinetic barrier is due to librational and hindered translational motions at the liquid surface, and that the lack of an isotope effect is due to competing energetic and entropic factors. The implications of these results for cloud and aerosol particles in the atmosphere are discussed.
\end{abstract}

\section{Introduction}

The evaporation and condensation rates of liquid water are of fundamental importance to many chemical, biological, and atmospheric processes. In particular, the formation and growth rates of cloud and aerosol particles are, in principle, sensitive to both kinetic and thermodynamic variables (Winkler et al., 2004). Clouds and aerosols have a cooling effect on the earth's atmosphere due to scattering of solar radiation, although some aerosols (black carbon) have a warming effect (IPCC, 2007). Current cloud models vary widely in their predictions for the radiative effects of anthropogenic emissions that affect the number and composition of parti-

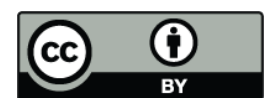

Correspondence to: R. C. Cohen (rccohen@berkeley.edu) cles on which cloud droplets condense (CCN) (IPCC, 2007; Laaksonen et al., 2005; McComiskey and Feingold, 2008; Lohmann et al., 2007). This variation is in part due to differing values for water evaporation and condensation kinetics and their relation to particle growth rates in these models (Laaksonen et al., 2005). Direct measurements of the microscopic rates of evaporation and condensation of pure water vary over three orders of magnitude, although recent measurements have narrowed the range to between $0.05-1$ times the gas kinetic limit (Eames et al., 1997; Marek and Straub, 2001; Davidovits et al., 2006). Some of the variation in older literature is likely due to impurities in or on the surface of the water samples used in the experiments; we note this fact hints that impurities will be important determinants of evaporation and condensation rates in mixed systems, a notion supported by field measurements of droplet growth rates (Feingold and Chuang, 2002; Ruehl et al., 2008). It is generally accepted that condensation and evaporation occurring faster than $10 \%$ of the gas kinetic limit results in thermodynamic control over droplet growth while slower rates result in kinetic control over these growth rates (Chuang et al., 1997; Laaksonen et al., 2005).

The maximum condensation rate of a gas is generally expressed via the Hertz-Knudsen equation, derived from gas kinetic theory (Eames et al., 1997),

$J_{c, \max }=\frac{p}{\sqrt{2 \pi m k T}}$,

where $p$ is the vapor pressure above the liquid surface, $m$ is the molecular mass, $K$ is Boltzmann's constant, and $T$ is the temperature. At equilibrium, the evaporation and condensation rates are equal; therefore the maximum evaporation rate can be expressed as

$J_{c, \max }=J_{e \cdot \max }=\frac{p_{\mathrm{sat}}}{\sqrt{2 \pi m k T}}$,

Published by Copernicus Publications on behalf of the European Geosciences Union. 


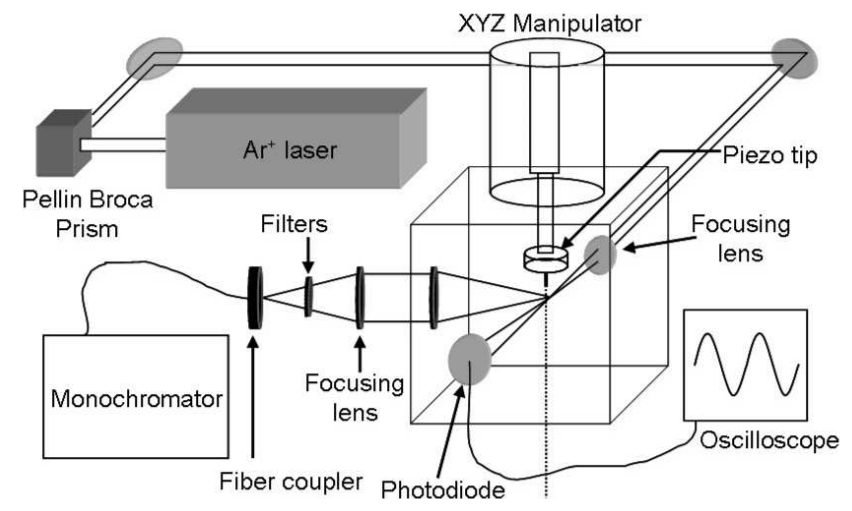

Fig. 1. Experimental apparatus. The droplet train is positioned onto the focal point of the $514.5 \mathrm{~nm}$ line of the $\mathrm{Ar}^{+}$laser with an XYZ manipulator. Raman scatter is collected at 90 degrees into a fiber coupler and routed to a monochromator (f/6.5) with a liquid nitrogen cooled CCD detector. A photodiode monitors the laser light attenuation after passing through the droplet train and is used in conjunction with an oscilloscope to ensure that uniform droplets are being produced. Spectra of the droplets as a function of time are taken by sampling at multiple points along the droplet stream, corresponding to liquid temperatures between $295 \mathrm{~K}$ and 255-260 K.

where $p_{\text {sat }}$ is the saturation vapor pressure. Since the activity of the pure liquid is unity, this expression for the evaporation rate holds at all vapor pressures. However, not all substances evaporate at the maximum rate (McFeely and Somorjai, 1972; Eames et al., 1997). Deviations from the maximum rate are treated by introducing the evaporation coefficient $\left(\gamma_{e}\right)$ and the condensation coefficient, alternatively referred to as the mass accommodation coefficient $\left(\alpha_{m}\right)$ :

$J_{e . \mathrm{obs}}=\gamma_{e} J_{e . \max }=\frac{\gamma_{e} p_{\mathrm{sat}}}{\sqrt{2 \pi m k T}}$

$J_{c . \mathrm{obs}}=\alpha_{m} J_{c . \max }=\frac{\alpha_{m} p}{\sqrt{2 \pi m k T}}$

The evaporation and mass accommodation coefficients, which have values between zero and one, must be equal due to detailed balance at equilibrium. The equality holds for non-equilibrium systems as long as the velocity distribution in the gas phase does not deviate significantly from a Boltzmann distribution, because a Boltzmann distribution is assumed in the derivation of Eq. (1). Indeed, theoretical treatments of condensation have shown little sensitivity to gas phase speeds selected from the Boltzmann distribution at room temperature, although the condensation probability lowers for very high speeds $(\sim 1000 \mathrm{~m} / \mathrm{s})$ (Chakraborty and Zachariah, 2008).

In addition to insuring the absence of impurities, measuring $\gamma_{e}$ for $\mathrm{H}_{2} \mathrm{O}$ is challenging because the high vapor pressure makes it difficult to observe evaporation or condensation in isolation without significant contributions from the oppos- ing term. In addition, knowledge of the liquid surface temperature is required, and evaporation results in cooling of the surface by as much as $3-4 \mathrm{~K}$ relative to the bulk (Ward and Stanga, 2001).

Our own experiments have made use of liquid jets and droplet streams with high vacuum $\left(10^{-4}\right.$ torr $)$ maintained around the fluid, such that evaporation occurs with negligible accompanying condensation. This greatly simplifies the interpretation as compared with many other recent experiments. The liquid jets and droplets also provide a renewing surface, minimizing contamination issues. Measurements of isotopic ratios in evaporation between 264 and $295 \mathrm{~K}$ showed that $\gamma_{e}<1$ and that it varied with the H/D ratio in the liquid (Cappa et al., 2005). Using Raman thermometry we derived a precise value of $\gamma_{e}$ from the temperature change associated evaporation of pure $\mathrm{H}_{2} \mathrm{O}$, yielding a value of $0.62 \pm 0.09$ over a temperature range of 245-295 K (Smith et al., 2006). We interpreted the results of our prior experiments using a transition-state theory (TST) model of liquid water evaporation (Cappa et al., 2007). The calculations indicated that the evaporation rate is primarily influenced by the intermolecular hindered translational and librational motions of molecules at the liquid surface.

Here we describe the extension of our previous studies to droplet train measurements of $\gamma_{e}$ of pure $\mathrm{D}_{2} \mathrm{O}$. These measurements serve as a further test of the microscopic theory of evaporation and of the reproducibility and precision of the methodology, providing a firm basis for future studies of the evaporation from mixtures of water with salts, oils or surfactants.

\section{Method}

Evaporation rates from liquid $\mathrm{D}_{2} \mathrm{O}$ in vacuum were determined by measuring the temperature change of evaporating droplets using Raman thermometry. The evaporation rate is deduced from the cooling rate and the well known heat of vaporization. The droplets were formed with a vibrating orifice aerosol generator (VOAG). The $\mathrm{D}_{2} \mathrm{O}$ used in this study was obtained from Cambridge Isotope Laboratories, with a stated purity of $99.9 \%$.

The Raman spectroscopy apparatus has been described in detail previously (Smith et al., 2006) and a schematic is given in Fig. 1. Briefly, a syringe pump (Teledyne ISCO Model 260D) is used to force the liquid through a fused silica orifice (2.5-4 $\mu$ m radius) mounted on a piezoelectric ceramic. The silica orifice is generated by pulling $100 \mu \mathrm{m}$ ID silica tubing to the desired size with a commercial $\mathrm{CO}_{2}$ laser micropipette puller. The piezo is driven with a $0-20 \mathrm{~V}$ square wave at 200 $1000 \mathrm{kHz}$ to generate a uniform droplet train with a spread in radius of less than $0.1 \mu \mathrm{m}$ (Sayer et al., 2003). The radii of the droplets produced is calculated from the liquid flowrate and the oscillation frequency (Smith et al., 2006). 
The VOAG is mounted on a bellows attached to the top of a $7 \mathrm{~cm}$ cubical vacuum chamber pumped by a $110 \mathrm{l} / \mathrm{s}$ turbomolecular pump. The VOAG as attached to an XYZ manipulator to allow positioning of the droplet stream. Pressures in the chamber during experiments were lower than $5 \times 10^{-4}$ torr. At these pressures, heat transfer from the walls of the chamber to the droplets is negligible. Viewports on the chamber allow the introduction of the $514.5 \mathrm{~nm}$ line from an argon ion laser operating at $\sim 250 \mathrm{~mW}$ or less, which is focused onto the droplet train. The laser power is sufficiently low to avoid heating the droplets or otherwise affecting their evaporation (Sayer et al., 2003; Smith and Baker, 1981). Raman scatter from the droplets is filtered and collected at 90 degrees through a fiber-optic cable and routed to a spectrometer with a liquid nitrogen cooled CCD camera. After the droplets leave the interaction volume, they enter a liquid nitrogen trap located $\sim 50 \mathrm{~cm}$ from the nozzle. To ensure that the droplets are uniform in size, a photodiode is placed in the path of the laser, after it has crossed the droplet train. The photodiode signal and the modulation frequency are monitored with an oscilloscope. As a droplet passes through the laser beam, there is a dip in signal on the photodiode; the oscillation frequency is tuned until the signal is sinusoidal in nature, indicating the formation of uniform droplets as described above.

The OD-stretching region of the Raman spectrum (2150$2800 \mathrm{~cm}^{-1}$ ) is used to determine the temperature of the droplets in a manner similar to that reported by Smith et al. (2006). Calibration curves were collected using both the thermostated nozzle technique described by Smith et al., and by measuring the total Raman scatter from liquid $\mathrm{D}_{2} \mathrm{O}$ in a cuvette over a similar temperature range $\left(0-50^{\circ} \mathrm{C}\right)$. The cuvette method was found to be more consistent $(<2 \%$ deviation) compared to the jet method ( $\sim 5 \%$ deviation) and has the additional advantage of requiring significantly smaller amounts of liquid. Calibrations taken using the cuvette method were used for the bulk of the data. Examples of the spectra used to generate the temperature calibrations are shown in Fig. 2a, and Fig. 2b shows one of the calibration curves.

Measurements were taken as a function of distance from the VOAG nozzle, which we converted to the residence time in the vacuum using the velocity of the droplet train. This velocity is calculated from the liquid flowrate and the orifice size. As described previously, the orifice size is determined by measuring the liquid jet diameter immediately after the nozzle using Mie scattering with the VOAG turned off (Cappa et al., 2005). The initial temperature of the droplets was determined by collecting the Raman spectrum of the droplet train in ambient air, where evaporative cooling is minimal (Smith et al., 2006).
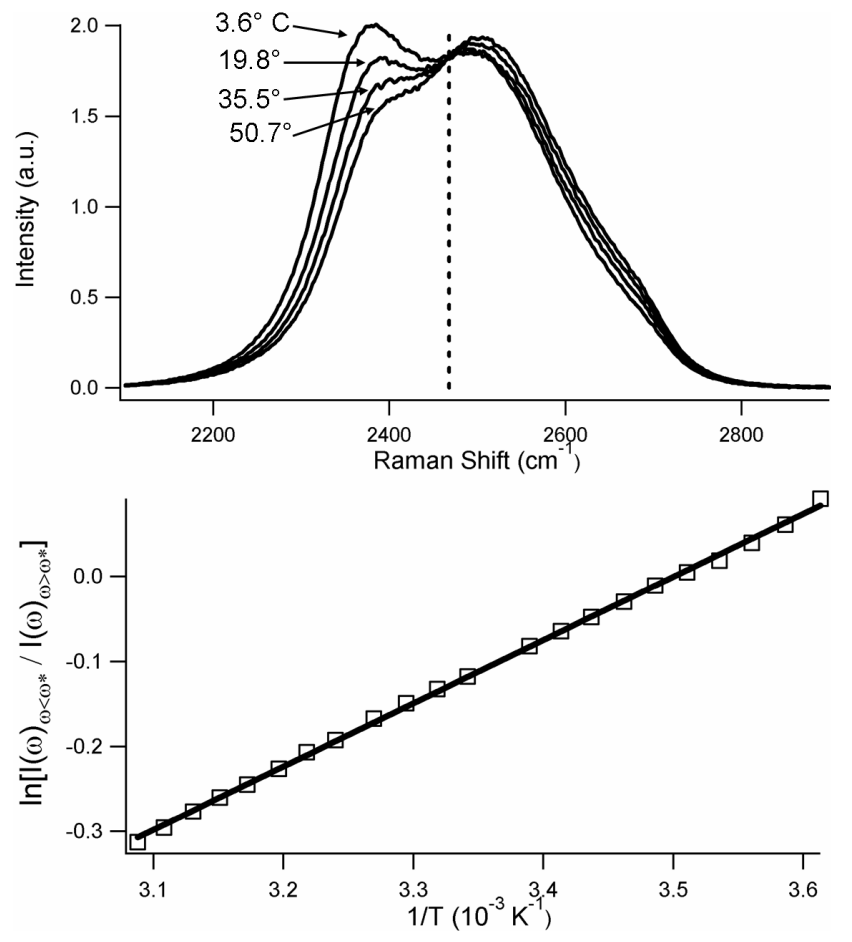

Fig. 2. (a) Representative spectra used to generate one of the temperature calibration curves. The dashed line shows the frequency $\omega^{*}=2468 \mathrm{~cm}^{-1}$ where the spectra were split. The full curve is constructed from spectra of liquid $\mathrm{D}_{2} \mathrm{O}$ at 22 different temperatures between $3.6^{\circ} \mathrm{C}$ and $50.7^{\circ} \mathrm{C}$. (b) Representative temperature calibration curve. $R^{2}=0.9992$.

\section{Results and analysis}

Measurements for eight different droplet sizes with radii in the range between $5.3 \mu \mathrm{m}$ and $8.1 \mu \mathrm{m}$ were performed. Data were collected as a function of residence time in the vacuum chamber for maximum times ranging from $435 \mu$ s to $1117 \mu \mathrm{s}$, with most around $600 \mu \mathrm{s}$. This corresponds to a temperature range from $295 \mathrm{~K}$ to as low as $255 \mathrm{~K}$. That our measurements are taken in the free evaporation regime was verified by calculating the average number of collisions experienced by an evaporating molecule as it leaves the droplet surface and reaches an infinite distance:

$$
\begin{aligned}
& N_{\text {coll }}\left(r_{0}, T\right)=\int_{r_{0}}^{\infty} \frac{d r}{\lambda(r, T)}=\sqrt{2} \pi d_{\text {coll }}^{2} n\left(r_{0}\right) r_{0}^{2} \int_{r_{0}}^{\infty} \frac{d r}{r^{2}} \\
& =\frac{r_{0}}{\lambda\left(r_{0}, T\right)},
\end{aligned}
$$

where $r_{0}$ is the droplet radius, $\lambda\left(r_{0}, T\right)=\left[\sqrt{2} \pi d_{\text {coll }} n(r)\right]^{-1}$ is the mean free path of the vapor, and $d_{\text {coll }}$ is the collision diameter $\left(2.6 \times 10^{-10} \mathrm{~m}\right)$. For $\mathrm{D}_{2} \mathrm{O}$, the vapor pressure predicted at a distance of $1 \mathrm{~mm}$ from the nozzle, where the first data point is taken, is $\sim 7.5$ torr. This corresponds to a mean free path of $\sim 12 \mu \mathrm{m}$; thus molecules evaporating 


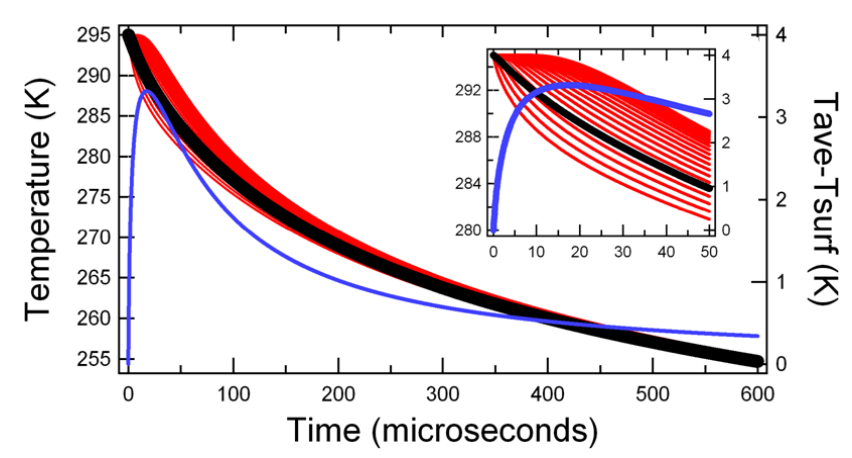

Fig. 3. Representative model output for a $6.65 \mu \mathrm{m}$ radius droplet with $\gamma_{e}$ of 0.57 . The black line is the volume-averaged temperature, the blue line is the magnitude of the thermal gradient within the droplet (the difference between the outer shell temperature and the volume-averaged temperature), and the red lines are the temperatures of each shell. Absolute temperature is on the left axis and the magnitude of the temperature difference is on the right axis. The inset is an enlarged image of the first $50 \mu$ s to depict the shell temperatures more clearly. The volume-averaged temperature (black line) is the output that is fit to the experimental data. Note that the thermal gradient quickly drops below $1 \mathrm{~K}$.

from droplets with radii less than $12 \mu \mathrm{m}$ experience less than one collision in the vapor phase on average and condensation may be neglected (Smith et al., 2006).

We model the observed cooling numerically in the same manner as in our study of $\mathrm{H}_{2} \mathrm{O}$ (Smith et al., 2006). We divide the droplet into concentric spherical shells, and considering evaporation from the outermost shell. Using Eq. (3), the cooling rate of the outermost shell is expressed as

$\frac{d T}{d t}=-\gamma_{e} A \frac{p_{\mathrm{sat}}}{\sqrt{2 \pi m k T}} \frac{\Delta H_{\mathrm{vap}}}{C_{p} \rho V_{s}}$,

where $A$ is the surface area of the outermost shell $\left(=4 \pi r_{0}^{2}\right)$, $p_{\text {sat }}$ is the satsuration vapor pressure, $m$ is the molecular mass, $K$ is Boltzmann's constant, $T$ is the temperature of the outermost shell, $\Delta H_{\mathrm{vap}}$ is the enthalpy of vaporization $\left(45.7 \mathrm{~kJ} / \mathrm{mol}\right.$ for $\left.\mathrm{D}_{2} \mathrm{O}\right), C_{p}$ is the specific heat capacity $\left(4.704 \mathrm{~kJ} / \mathrm{kg}^{*} \mathrm{~K}\right.$ for $\left.\mathrm{D}_{2} \mathrm{O}\right), \rho$ is the density, and $V_{s}$ is the volume of the outermost shell. This simplifies to

$$
\frac{d T}{d t}=-\gamma_{e} \frac{p_{\mathrm{sat}}}{\sqrt{2 \pi m k T}} \frac{\Delta H_{\mathrm{vap}}}{C_{p}} \frac{3 r_{0}^{2}}{\left(r_{0}^{3}-r_{1}^{3}\right) \rho_{1}},
$$

where $r_{0}$ and $r_{1}$ are the outer and inner radii of the outermost shell of the droplet. The only tunable parameter is $\gamma_{e}$. Thermal diffusion between adjacent shells is modeled as

$\frac{d Q}{d t}=-\kappa A \frac{d T}{d r}$,

where $\kappa$ is the thermal conductivity $(0.595 \mathrm{~W} / \mathrm{m} * \mathrm{~K}$ at $298 \mathrm{~K}$ for $\left.\mathrm{D}_{2} \mathrm{O}\right), \mathrm{A}$ is the surface area of the shell and $\mathrm{dT} / \mathrm{dr}$ is the temperature difference between the two adjacent shells; we do not assume instantaneous thermal equilibrium. As the outermost shell evaporates, mass loss due to evaporation is taken into account and the droplet and all the shells are resized accordingly at each time step $\left(10^{-10} \mathrm{~s}\right)$. The droplet radius typically decreases by $\sim 5 \%$ over the duration of a measurement. The temperature gradient and the volumeaveraged temperature of the entire droplet are calculated at each time step. As the entire droplet resides within the laser focal volume, we interpret the observed temperature as the volume-averaged temperature. We determine $\gamma_{e}$ by fitting the observations to the model represented by Eq. (7). We have considered three variations on the form of $\gamma_{e}$, one where $\gamma_{e}$ is a constant with temperature and two where $\gamma_{e}$ is allowed to vary with temperature.

An example calculation is shown in Fig. 3. The figure shows the calculated temperatures for each of 20 spherical shells as a function of time, as well as the volume-averaged temperature for the entire $6.65 \mu \mathrm{m}$ droplet. The magnitude of the surface-bulk temperature difference in the droplet, defined as the difference between the outer shell temperature and the volume-averaged temperature of the droplet, is also shown. The maximum difference is $3 \mathrm{~K}$, which occurs during the first $100 \mu \mathrm{s}$ and thereafter drops below $1 \mathrm{~K}$. Tests of the numerical accuracy of the calculations show that 20 shells are sufficient to converge the calculations.

There are a few assumptions made in the modeling procedure, but these have little effect on the model results. First, we assume that there is no re-condensation, only evaporation. There is, however, a small amount of condensation resulting from evaporating molecules impinging on adjacent droplets in the droplet train. This effect has been quantified previously and leads to an underestimate of $\gamma_{e}$ of less than 0.01 (Smith et al., 2006). Second, we assume that the liquid surface in our vacuum system can be directly compared with the liquid surface in an equilibrium system. On sufficiently short timescales, it is possible that the rapid evaporation in the absence of condensation could affect the liquid surface structure and thus the evaporation dynamics. However, if one assumes that $\gamma_{e}=1$ (i.e. the maximum evaporation rate) and considers evaporation from a $1 \mathrm{~nm}$ square patch of liquid surface, which is larger than the water-water correlation length (Head-Gordon and Hura, 2002), Eq. (3) yields an evaporation rate of one evaporating molecule every 10 ns. (Note: We presented a similar argument in (Smith et al., 2006) but incorrectly reported a $10 \mathrm{~nm}$ square instead of a $1 \mathrm{~nm}$ square.) Molecular simulations suggest that the timescale for reorganization of the liquid water surface is on the order of a few picoseconds (Garrett et al., 2006). Therefore, evaporation events are too rare to perturb the liquid surface structure, and the surface should be the same under vacuum as it is in equilibrium. Lastly, the model treats some parameters as constant, such as density and thermal conductivity, which in fact vary with temperature. For completeness, a temperature-dependent equation for the density of $\mathrm{D}_{2} \mathrm{O}$ from Kell (Kell, 1967) and an empirical temperatureand density-dependent equation for the thermal conductivity 
of $\mathrm{D}_{2} \mathrm{O}$ from the International Association for the Properties of Water and Steam (Kestin, 2007) were both incorporated into the model. The inclusion of these temperature dependent values changed $\gamma_{e}$ by less than $0.1 \%$.

We tune $\gamma_{e}$ in this model to fit observations of a $6.65 \mu \mathrm{m}$ droplet train, shown in Fig. 4a. For this particular experiment, a best fit (assuming zero temperature dependence of $\gamma_{e}$ ) is obtained for $\gamma_{e}=0.51$. Eight different droplet sizes were measured. The average evaporation coefficient derived $(95 \%$ confidence interval) is $0.57 \pm 0.06$ where we have assumed no temperature dependence.

The reported error is primarily experimental and is likely associated with determining the temperature from the Raman spectrum; we are only able to obtain the temperature with a precision of $\pm 2 \mathrm{~K}$. This limitation arises from the calibration curves used. Other possible sources of experimental error are small; shape oscillations in the droplets produced by the VOAG are expected to decay after a few microseconds, and are therefore negligible on the $>500 \mu$ s timescale of the measurements (Weierstall et al., 2008; Devarakonda et al., 1998). Other effects of the droplet production, such as rotation of the droplets, should be minimal and are not expected to affect the evaporation.

The theoretical cooling curve for $\gamma_{e}=1$, also shown in Fig. 4a, yields significantly more cooling for a given interaction time than was observed. For comparison, we have also included the model results using the temperature dependent $\gamma_{e}$ from Li et al, which increases from 0.17 at $295 \mathrm{~K}$ to 0.32 at $258 \mathrm{~K}$ (Li et al., 2001). This predicts significantly less cooling than was observed. We have also fit two different temperature dependent functions to this data set, as shown in Fig. 4b. These functions represent the maximum positive and negative temperature dependences that are still consistent with the observations. First, we use the functional form of the temperature dependence described by $\mathrm{Li}$ et al., but adjusted to give a larger $\gamma_{e}$ at lower temperature than that measured by $\mathrm{Li}$ et al. This yields results consistent with our observations if $\gamma_{e}$ is equal to 0.4 at $295 \mathrm{~K}$ and to 0.6 at $258 \mathrm{~K}$. Second, we assume an exponential temperature dependence for $\gamma_{e}$ of the form $\gamma_{e}=\rho \exp \left(-E_{a} / R T\right)$, where $\rho$ was constrained to be $\leq 1$. The maximum $\mathrm{E}_{a}$ for this case that is still consistent with observations is $1.8 \mathrm{~kJ} / \mathrm{mol}$, corresponding to a $\gamma_{e}$ of 0.48 at $295 \mathrm{~K}$ and 0.43 at $258 \mathrm{~K}$.

\section{Discussion}

The observed value of $\gamma_{e}$ for $\mathrm{D}_{2} \mathrm{O}$ is smaller than unity. The value is nearly identical to that obtained recently for $\mathrm{H}_{2} \mathrm{O}$ $(0.62 \pm 0.09)$ (Smith et al., 2006). The TST model study by Cappa et al. predicts that $\mathrm{H}_{2} \mathrm{O}$ and $\mathrm{D}_{2} \mathrm{O}$ would have similar values of $\gamma_{e}$, based on calculations of the absolute evaporation rates of the different isotopes as a function of deuterium mole fraction in the liquid (Fig. 5 of that study), although the relative evaporation rates of the pure liquids was not explic-
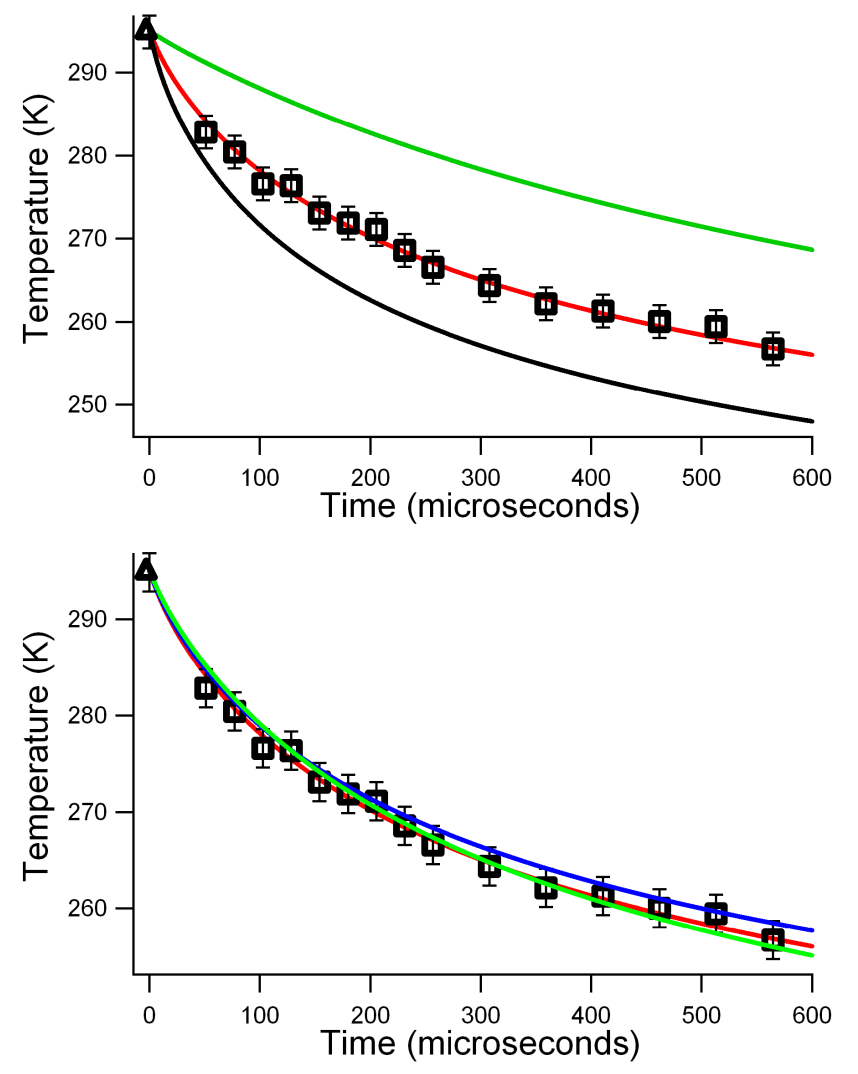

Fig. 4. (a) Experimental data for a droplet size of $6.65 \mu \mathrm{m}$ radius, shown with the model fit (red line) to $\gamma_{e}=0.51$. The triangle represents the "time zero" data point taken in ambient air, and the squares represent the data taken under vacuum. The black line shows the model output for $\gamma_{e}=1$. The green line represents the predicted cooling using the temperature dependent $\gamma_{e}$ from $\mathrm{Li}$ et al. (b) The same data, shown with the temperature independent fit for $\gamma_{e}=0.51$ (red line), a temperature dependent fit with the functional form from $\mathrm{Li}$ et al. (green line) where $\gamma_{e}$ increases from 0.4 at $295 \mathrm{~K}$ to 0.6 at $258 \mathrm{~K}$, and an exponential temperature dependent fit (blue line) corresponding to an activation energy of $1.8 \mathrm{~kJ} / \mathrm{mol}$. This exponential function is constrained to give $\gamma_{e} \leq 1$ for all temperatures. The exponential fit gives $\gamma_{e}=0.48$ at $295 \mathrm{~K}$ and $\gamma_{e}=0.43$ at $258 \mathrm{~K}$. Both temperature-dependent fits were tuned to yield the strongest temperature dependence that agrees with the observations within experimental error.

itly calculated (Cappa et al., 2007). To determine whether an isotope effect is predicted, we calculated the ratio of $\gamma_{e}$ for pure $\mathrm{H}_{2} \mathrm{O}$ to that for pure $\mathrm{D}_{2} \mathrm{O}$ using the following equation adapted from Cappa et al. (2007):

$r_{\gamma}=\frac{\gamma_{e, H}}{\gamma_{e, D}}=\frac{J_{e, \max }^{D}}{J_{e, \max }^{H}} \frac{Q_{H}^{*}}{Q_{D}^{*}} \frac{Q_{s}^{D}}{Q_{s}^{H}} e^{-\Delta E_{a} / k T}$,

where $J_{e, \max }$ is the maximum evaporation rate from Eq. (2), $Q^{*}$ and $Q_{s}$ are the partition functions of the transition state and the liquid surface species, respectively, and $\Delta E_{a}$ is the difference in activation energies between the two isotopes 
(Cappa et al., 2007). The sub- and superscripts $\mathrm{H}$ and D refer to $\mathrm{H}_{2} \mathrm{O}$ and $\mathrm{D}_{2} \mathrm{O}$, respectively. By calculating the ratio $\mathrm{r}_{\gamma}$ we avoid several assumptions associated with calculating the absolute evaporation rates of the isotopes, such as knowledge of the transition state area and the absolute magnitude of the activation energy (Cappa et al., 2007).

The observed ratio from experiment, $\mathrm{r}_{\gamma, \exp }$, is $1.09 \pm 0.18$. The calculated ratio $\mathbf{r}_{\gamma}$ from Eq. (9) was found to vary between 0.90 and 1.08 at $295 \mathrm{~K}$, depending on the specific choices of $Q^{*}, Q_{s}$, and $\Delta E_{a}$ given in Cappa et al. (Cappa et al., 2007). The smallest isotope effect $\left(\mathrm{r}_{\gamma}=1.02\right)$ was found using the values derived from the "scaled" surface frequencies. These intermolecular translational and librational frequencies of the liquid surface species were scaled by a factor of $\sqrt{3 / 4}$ relative to the bulk frequencies to better approximate the looser binding at the surface (Smith et al., 2003). At $265 \mathrm{~K}, \mathrm{r}_{\gamma}$ varies between 0.84 and 0.99 , with the scaled frequencies yielding $\mathrm{r}_{\gamma}=0.94$. This difference implies a very weak temperature dependence to the relative values of $\gamma_{e}$ for the two isotopes. We note that a value of $r_{\gamma}$ less than unity implies that $\mathrm{D}_{2} \mathrm{O}$ has a higher evaporation coefficient than $\mathrm{H}_{2} \mathrm{O}$, but does not imply that it has a higher evaporation rate; the maximum theoretical rates for the two species are different according to Eq. (2). The small isotope effect is due to a competing effect between the partition function ratios in the exponential pre-factor (entropy) and the activation energy difference in the exponential. The results of our previous study of the evaporation of isotopic mixtures and the calculations by Cappa et al. suggests that these effects only balance each other for the pure liquids, and that $\gamma_{e}$ for $\mathrm{H}_{2} \mathrm{O}$ and $\mathrm{D}_{2} \mathrm{O}$ in different isotopic mixtures can differ by as much as a factor of 3 (Cappaet al., 2005; Cappa et al., 2007). It is important to note that the hindered translational frequencies for $\mathrm{D}_{2} \mathrm{O}$ are taken to be smaller than those of $\mathrm{H}_{2} \mathrm{O}$ when comparing the pure solutions, as this plays an important role in determining $\mathrm{r}_{\gamma}$. The activation energy for $\mathrm{D}_{2} \mathrm{O}$ evaporation is predicted to be $\sim 2 \mathrm{~kJ} / \mathrm{mol}$ higher than that for $\mathrm{H}_{2} \mathrm{O}$, which is approximately the difference expected from zero point energy effects. Therefore, this assessment remains valid if both $\mathrm{H}_{2} \mathrm{O}$ and $\mathrm{D}_{2} \mathrm{O}$ have no energetic barrier to evaporation and the kinetic limit results entirely from entropic effects; however, a small energetic barrier is certainly possible. Recent measurements by Ward and Stanga showed a small $\left(\sim 8^{\circ} \mathrm{C}\right)$ temperature discontinuity between evaporating liquid $\mathrm{H}_{2} \mathrm{O}$ and the vapor (Ward and Stanga, 2001). Assuming that the higher temperature in the vapor is due to an energetic barrier to evaporation, and using the specific heat capacity of the vapor $\left(\mathrm{C}_{p}=37.47 \mathrm{~J} \mathrm{~mol}^{-1} \mathrm{~K}^{-1}\right)$, the barrier size is predicted to be very small $(\sim 300 \mathrm{~J} / \mathrm{mol})$ for $\mathrm{H}_{2} \mathrm{O}$. Our data provide a weak constraint on the size of the barrier. The analysis above suggested the energetic barrier is less than $1.8 \mathrm{~kJ} / \mathrm{mol}$ for $\mathrm{D}_{2} \mathrm{O}$.

The apparent lack of a temperature dependence to $\gamma_{e}$ observed in this study and that of Smith et al. (Smith et al., 2006) may appear to be in contrast to an earlier study by
Cappa et al. (Cappa et al., 2005) wherein a stronger temperature dependence to $\gamma_{e}$ was suggested. As noted in that work, and discussed in a later publication (Cappa et al., 2007), the prediction involved several assumptions and high uncertainty. However, the relative evaporation rates of the isotopic species in the mixtures measured in that study are expected to display a temperature dependence.

Our measurements of $\gamma_{e}$ for $\mathrm{D}_{2} \mathrm{O}$ and $\mathrm{H}_{2} \mathrm{O}$ can be compared with other recent measurements. Our value of $\gamma_{e}$ falls within the range of, but has much higher precision than, recent measurements by Winkler et al. (Winkler et al., 2004; Winkler et al., 2006) and Voigtlander et al. (Voigtlander et al., 2007), both of which were condensation studies. Winkler et al. found $\gamma_{e}$ to be between $0.8-1.0$ for temperatures between 250 and $270 \mathrm{~K}$ and $0.4-1.0$ for temperatures between 270 and $290 \mathrm{~K}$, although values higher than 1 (up to 10 in the case of $290 \mathrm{~K}$ ) were within error for these measurements. Values larger than unity are not physically meaningful. Voigtlander et al. found $\gamma_{e}$ to be between 0.3 and 1 for uptake on $\mathrm{NaCl}$ particles. $\mathrm{Li}$ et al. determined that $\gamma_{e}$ has an inverse temperature dependence, with $\gamma_{e}$ increasing from $0.17 \pm 0.03$ at $285 \mathrm{~K}$ to $0.32 \pm 0.04$ at $258 \mathrm{~K}$ (Li et al., 2001). Similarly, a group from the Polish Academy of Sciences (Jakubczyk et al., 2007; Zientara et al., 2008) observed $\gamma_{e}$ to increase from 0.13 at $293.1 \mathrm{~K}$ to 0.18 at $273.1 \mathrm{~K}$. It is important to note that in all of these studies, the $\gamma_{e}$ values determined are higher than the 0.1 threshold below which cloud formation becomes kinetically controlled. While attempts have been made to reconcile some of these experiments (Davidovits et al., 2004) there is not yet a satisfactory explanation for the observed differences. A recent study by Fukuta and Myers (Fukuta and Myers, 2007) highlights the "moving boundary effect" which can occur during modeling of evaporation or condensation. They report that incorrectly accounting for the shifting liquid-vapor boundary as a droplet grows or shrinks can lead to errors in the calculated evaporation or condensation coefficients as large as several percent. In our case, the droplet radius shrinks by $\sim 5 \%$ over the duration of a measurement, but the moving boundary effect is explicitly accounted for within the model by resizing the droplet at every timestep. Zientara et al. argue that even larger corrections may be necessary in many cases due to thermal effusion near the droplet surface (Zientara et al., 2008). Many models of condensation and evaporation treat the vapor using the framework of diffusion, but Zientara et al. argue that at distances below the mean free path of the vapor, a droplet must be modeled as evaporating and condensing through vacuum. In certain cases, while the droplet would be considered to be quasi-stationary in the diffusion framework, thermal effusion near the surface can significantly change the temperature of the surface of the droplet from what would be predicted by diffusion, dependent on the different timescales of various experiments (Zientara et al., 2008). In our experiments, the vapor is negligible and our model explicitly accounts for the cooling of the surface of the droplets. Therefore such a 
temperature jump cannot explain the difference between our measured values and those of $\mathrm{Li}$ et al. (2001) and Zientara et al. (2008).

In a previous work (Cappa et al., 2005) we discussed a possible source of discrepancy between our experiments and those of Li et al. (2001), claiming that our formulations of $\gamma_{e}$ and $\alpha_{m}$ are different, with $\gamma_{e} \sim\left(1-\alpha_{m}\right)$. We now recognize that this prior analysis was incorrect and resulted from equating two different rates in formulations of the evaporation and mass accommodation processes which are not equivalent. Reanalysis of the different formulations of $\gamma_{e}$ and $\alpha_{m}$ indicates that the formulations are equivalent, and consistent with the definitions in Eqs. (3) and (4) of the present manuscript.

Our reported value of $\gamma_{e}=0.57$ for $\mathrm{D}_{2} \mathrm{O}$ provides support for our previous results for $\mathrm{H}_{2} \mathrm{O}$, confirming that $\gamma_{e}$ for pure $\mathrm{H}_{2} \mathrm{O}$ is not small enough to have a significant impact on formation rates of cloud droplets in the atmosphere (Smith et al., 2006). There are other effects, however, that could lower water uptake rates on ambient $\mathrm{CCN}$, which are not pure $\mathrm{H}_{2} \mathrm{O}$ or $\mathrm{D}_{2} \mathrm{O}$, such as the presence of concentrated solutes or surfactant coatings on the droplets. For example, the effects of dissolved salts on the vapor pressure of liquid water have been extensively studied (MacMullin, 1969; Hornung and Giauque, 1955; Horita et al., 1992; Shmulovich et al., 1999), but the kinetic effects on the evaporation coefficient are unknown. It has been argued that surface active solutes can lower the evaporation rate considerably, possibly leading to large changes in $\gamma_{e}$ (Marek and Straub, 2001). Many studies of the effect of surfactants on water evaporation indicate a dramatic lowering of the evaporation rate upon sufficient surface coverage by surfactant molecules (Lunkenheimer and Zembala, 1997; Rusdi and Moroi, 2004; Seaver et al., 1992). Additionally, recent molecular dynamics (MD) simulations of condensation onto an aerosol particle coated with organics show a significantly lowered condensation probability (Chakraborty and Zachariah, 2008). Given that CCN are often composed of inorganic solutes as well as of organics which might partition to the surface, measurements of $\gamma_{e}$ on these more complex mixtures will be important to improving microscopic models of cloud droplet growth rates, number and size. Indeed, a recent study sampling atmospheric aerosol from several different sites found that cloud droplet growth rates were often consistent with values of $\gamma_{e}$ less than 0.1 (Ruehl et al., 2008), providing additional motivation for continued development of methods capable of precise determination of $\gamma_{e}$.

\section{Conclusions}

We have determined the evaporation coefficient of $\mathrm{D}_{2} \mathrm{O}$ to be $0.57 \pm 0.06$ using Raman thermometry measurements on droplets undergoing evaporation in the absence of condensation. This value is the same, within experimental error, as that previously measured for $\mathrm{H}_{2} \mathrm{O}$ (Smith et al., 2006). Thus, $\gamma_{e}$ for pure water is less than unity, but is not small enough to have a significant impact on models for cloud formation and aerosol growth rates. A TST model for water evaporation (Cappa et al., 2007) is consistent with both the $\mathrm{H}_{2} \mathrm{O}$ and $\mathrm{D}_{2} \mathrm{O}$ observations and indicates that the lack of an isotope effect is due to competing energetic and entropic effects. Further understanding of the source and magnitude of these effects and how they are affected by the presence of salts, oils and surfactants will likely be important to understanding evaporation and condensation in mixed phase systems.

Acknowledgements. This work was supported by NSF ATM 0639847 and the Director, Office of Science, Office of Basic Energy Sciences, of the US Department of Energy under Contract No. DE-AC02-05CH11231. W. S. Drisdell thanks the Berkeley Atmospheric Science Center for a graduate student research fellowship.

Edited by: W. E. Asher

\section{References}

Cappa, C. D., Drisdell, W. S., Smith, J. D., Saykally, R. J., and Cohen, R. C.: Isotope fractionation of water during evaporation without condensation, J. Phys. Chem. B, 109, 24 391-24 400, 2005.

Cappa, C. D., Smith, J. D., Drisdell, W. S., Saykally, R. J., and Cohen, R. C.: Interpreting the h/d isotope fractionation of liquid water during evaporation without condensation, J. Phys. Chem. C, 111, 7011-7020, 2007.

Chakraborty, P. and Zachariah, M. R.: Sticking coefficient and processing of water vapor on organic-coated nanoaerosols, J. Phys. Chem. A, 112, 966-972, 2008.

Chuang, P. Y., Charlson, R. J., and Seinfeld, J. H.: Kinetic limitations on droplet formation in clouds, Nature, 390, 594-596, 1997.

Davidovits, P., Worsnop, D. R., Jayne, J. T., Kolb, C. E., Winkler, P., Vrtala, A., Wagner, P. E., Kulmala, M., Lehtinen, K. E. J., Vesala, T., and Mozurkewich, M.: Mass accommodation coefficient of water vapor on liquid water, Geophys. Res. Lett., 31, 2004.

Davidovits, P., Kolb, C. E., Williams, L. R., Jayne, J. T., and Worsnop, D. R.: Mass accommodation and chemical reactions at gas-liquid interfaces, Chem. Rev., 106, 1323-1354, 2006.

Devarakonda, V., Ray, A. K., Kaiser, T., and Schweiger, G.: Vibrating orifice droplet generator for studying fast processes associated with microdroplets, Aerosol Sci. Technol., 28, 531-547, 1998.

Eames, I. W., Marr, N. J., and Sabir, H.: The evaporation coefficient of water: A review, International Journal Of Heat And Mass Transfer, 40, 2963-2973, 1997.

Feingold, G. and Chuang, P. Y.: Analysis of the influence of filmforming compounds on droplet growth: Implications for cloud microphysical processes and climate, J. Atmos. Sci., 59, 20062018, 2002.

Fukuta, N. and Myers, M. N.: Simultaneous measurement of condensation and thermal accommodation coefficients for cloud droplet growth in due consideration of a new moving surfaceboundary effect, J. Atmos. Sci., 64, 955-968, 2007. 
Garrett, B. C., Schenter, G. K., and Morita, A.: Molecular simulations of the transport of molecules across the liquid/vapor interface of water, Chem. Rev., 106, 1355-1374, 2006.

Head-Gordon, T. and Hura, G.: Water structure from scattering experiments and simulation, Chem. Rev., 102, 2651-2669, 2002.

Horita, J., Wesolowski, D. J., and Cole, D. R.: The activitycomposition relationship of oxygen and hydrogen isotopes in aqueous salt solutions: I. Vapor-liquid water equilibration of single salt solutions from 50 to $100 \mathrm{c}$, Geochim. Cosmochim. Ac., 57, 2797-2817, 1992.

Hornung, E. W. and Giauque, W. F.: The vapor pressure of water over aqueous sulfuric acid at 25-degrees, J. Am. Chem. Soc., 77, 2744-2746, 1955.

Jakubczyk, D., Zientara, M., Kolwas, K., and Kolwas, M.: Temperature dependence of evaporation coefficient for water measured in droplets in nitrogen under atmospheric pressure, J. Atmos. Sci., 64, 996-1004, 2007.

Kell, G. S.: Precise representation of volume properties of water at 1 atmosphere, J. Chem. Eng. Data, 12, 66-69, 1967.

Kestin, J.: Revised release on viscosity and thermal conductivity of heavy water substance, International Association for the Properties of Water and Steam, 1-17, 2007.

Laaksonen, A., Vesala, T., Kulmala, M., Winkler, P. M., and Wagner, P. E.: Commentary on cloud modelling and the mass accommodation coefficient of water, Atmos. Chem. Phys., 5, 461-464, 2005 , http://www.atmos-chem-phys.net/5/461/2005/.

Li, Y. Q., Davidovits, P., Shi, Q., Jayne, J. T., Kolb, C. E., and Worsnop, D. R.: Mass and thermal accommodation coefficients of $\mathrm{h} 2 \mathrm{o}(\mathrm{g})$ on liquid water as a function of temperature, J. Phys. Chem. A, 105, 10627-10 634, 2001.

Lohmann, U., Quaas, J., Kinne, S., and Feichter, J.: Different approaches for constraining global climate models of the anthropogenic indirect aerosol effect, Bull. Am. Meteorol. Soc., 88, 243-249, 2007.

Lunkenheimer, K. and Zembala, M.: Attempts to study a water evaporation retardation by soluble surfactants, J. Colloid Interf. Sci., 188, 363-371, 1997.

MacMullin, R. B.: Algorithms for vapor pressure of water over aqueous solutions of salt and caustic soda, J. Electrochem. Soc., 116, 416-419, 1969.

Marek, R. and Straub, J.: Analysis of the evaporation coefficient and the condensation coefficient of water, Int. J. Heat Mass Tran., 44, 39-53, 2001.

McComiskey, A. and Feingold, G.: Quantifying error in the radiation forcing of the first aerosol indirect effect, Geophys. Res. Lett., 35, 2008.

McFeely, F. R. and Somorjai, G. A.: Studies of vaporization kinetics of hydrogen-bonded liquids, J. Phys. Chem., 76, 914-918, 1972.

Ruehl, C. R., Chuang, P. Y., and Nenes, A.: How quickly do cloud droplets form on atmospheric particles?, Atmos. Chem. Phys., 8, 1043, 2008.
Rusdi, M. and Moroi, Y.: Study on water evaporation through 1alkanol monolayers by the thermogravimetry method, J. Colloid Int. Sci., 272, 472-479, 2004.

Sayer, R. M., Gatherer, R. D. B., Gilham, R. J. J., and Reid, J. P.: Determination and validation of water droplet size distributions probed by cavity enhanced raman scattering, Phys. Chem. Chem. Phys., 5, 3732-3739, 2003.

Seaver, M., Peele, J. R., Manuccia, T. J., Rubel, G. O., and Ritchie, G.: Evaporation kinetics of ventilated waterdrops coated with octadecanol monolayers, J. Phys. Chem., 96, 6389-6394, 1992.

Shmulovich, K. I., Landwehr, D., Simon, K., and Heinrich, W.: Stable isotope fractionation between liquid and vapour in watersalt systems up to 600 degrees c, Chem. Geol., 157, 343-354, 1999.

Smith, J. A., Livingston, F. E., and George, S. M.: Isothermal desorption kinetics of crystalline h2o, (h2o)-o-18, and d2o ice multilayers, J. Phys. Chem. B, 107, 3871-3877, 2003.

Smith, J. D., Cappa, C. D., Drisdell, W. S., Cohen, R. C., and Saykally, R. J.: Raman thermometry measurements of free evaporation from liquid water droplets, J. Am. Chem. Soc., 128, 12 892-12 898, 2006.

Smith, R. C. and Baker, K. S.: Optical-properties of the clearest natural-waters (200-800 nm), Appl. Optics, 20, 177-184, 1981.

Voigtlander, J., Stratmann, F., Niedermeier, D., Wex, H., and Kiselev, A.: Mass accomodation coefficient of water: A combined computational fluid dynamics and experimental analysis, J. Geophys. Res., 112, D20208, doi:10.1029/2007JD008604, 2007.

Ward, C. A. and Stanga, D.: Interfacial conditions during evaporation or condensation of water, Phys. Rev. E, 6405, 051509, 2001.

Weierstall, U., Doak, R. B., Spence, J. C. H., Starodub, D., Shapiro, D., Kennedy, P., Warner, J., Hembree, G. G., Fromme, P., and Chapman, H. N.: Droplet streams for serial crystallography of proteins, Exp. Fluids, 44, 675-689, 2008.

Warner, J., Hembree, G. G., Fromme, P., and Chapman, H. N.: Droplet streams for serial crystallography of proteins, Exp. Fluids, 44, 675-689, 2008.

Winkler, P. M., Vrtala, A., Wagner, P. E., Kulmala, M., Lehtinen, K. E. J., and Vesala, T.: Mass and thermal accommodation during gas-liquid condensation of water, Phys. Rev. Lett., 93, 0757011-075701-4, 2004.

Winkler, P. M., Vrtala, A., Rudolf, R., Wagner, P. E., Riipinen, I., Vesala, T., Lehtinen, K. E. J., Viisanen, Y., and Kulmala, M.: Condensation of water vapor: Experimental determination of mass and thermal accommodation coefficients, J. Geophys. Res.-Atmos., 111, D19202, doi:10.1029/2006JD007194, 2006.

Zientara, M., Jakubczyk, D., Kolwas, K., and Kolwas, M.: Temperature dependence of the evaporation coefficient of water in air and nitrogen under atmospheric pressure: Study in water droplets, J. Phys. Chem. A, 112, 5152-5158, 2008. 